\title{
Use of population approach non-linear mixed effects models in the evaluation of biosimilarity of monoclonal antibodies
}

\author{
Joannes A. A. Reijers $^{1}$ • T. van Donge ${ }^{1}$ - F. M. L. Schepers ${ }^{1}$. J. Burggraaf ${ }^{1} \cdot$ J. Stevens $^{1}$
}

Received: 7 May 2016 / Accepted: 13 July 2016/Published online: 11 August 2016

(C) The Author(s) 2016. This article is published with open access at Springerlink.com

\begin{abstract}
Purpose Population pharmacokinetic analyses (PPK) have been used to establish bioequivalence for small molecules and some biologicals. We investigated whether PPK could also be useful in biosimilarity testing for monoclonal antibodies (MAbs).

Methods Data from a biosimilarity trial with two trastuzumab products were used to build population pharmacokinetic models. First, a combined model was developed and similarity between test and reference product was evaluated by performing a covariate analysis with trastuzumab drug product (test or reference) on all model parameters. Next, two separate models were developed, one for each drug product. The model structure and parameters were compared and evaluated for differences.

Results Drug product could not be identified as statistically significant covariate on any parameter in the combined model, and the addition of drug product as covariate did not improve the model fit. A similar structural model described both the test and reference data best. Only minor differences were found between the estimated parameters from these separate models.

Conclusions PPK can also be used to support a biosimilarity claim for a MAb. However, in contrast to the standard noncompartmental analysis, there is less experience with a PPK
\end{abstract}

Electronic supplementary material The online version of this article (doi:10.1007/s00228-016-2101-6) contains supplementary material, which is available to authorized users.

Joannes A. A. Reijers

info@chdr.nl

1 Centre for Human Drug Research (CHDR), Zernikedreef 8, 2333 CL Leiden, The Netherlands approach. Here, we describe two methods of how PPK can be incorporated in biosimilarity testing for complex therapeutics.

Keywords Biosimilarity · Population pharmacokinetic modelling · Pharmacokinetics · Biological · Trastuzumab · Non-linear kinetics

\section{Introduction}

During the past decades, many biotherapeutics have been marketed, mostly for use in the field of oncology and rheumatology. Although efficacious, high costs often limit the availability of these therapies or greatly burden the health care system. For example, treatment of a rheumatologic US patient with biologics costs on average $\$ 20,000$ to $\$ 30,000$ annually [1], and a single cycle of bevacizumab or other monoclonal antibody (MAb) can cost $\$ 2000$ or more [2]. In 2014, the top 20 in global medicinal product sales contained 10 biotherapeutics, generating revenues between 4.4 and 11.8 billion dollar each [3]. Because of the growing number of patent expirations for the original biotherapeutics, it is expected that research of biosimilars will increase.

A first requirement for registration of the novel compound is to establish pharmacokinetic 'biosimilarity'. Although the terminology slightly differs between the regulatory agencies, all agree on the basic concept of biosimilarity, which is that the novel ('test') compound should be highly similar to its originator ('reference') in terms of quality, efficacy and safety and that any remaining difference should be clinically insignificant [4-6].

Notwithstanding specific criteria for biotherapeutics, often, parts of guidelines for establishing bioequivalence- not biosimilarity - between chemically derived substances ('small molecules') are applied. These guidelines require that 
similarity should be demonstrated for key pharmacokinetic parameters, most commonly area under the concentrationtime curve (AUC) and maximum concentration $\left(C_{\max }\right)$, based on predefined acceptance limits at the highest dose level used. According to an evaluation by the World Health Organisation, studies proving biosimilarity generally use the 80-125\% equivalence range due to lack of specific acceptance criteria for biotherapeutics [4].

Although it is widely recognised that a non-compartmental analysis (NCA) is less appropriate when dealing with complex pharmacokinetics, it is still the most commonly used analytical method for demonstrating biosimilarity. Mentré's group has extensively studied the use of population pharmacokinetic techniques in bioequivalence testing and found that it yielded similar results, with the modelling approach leading to a better understanding of the underlying biological system and the NCA being a relatively easy approach that does not require modelling and whose results can be used in a statistical analysis. The same was found for two biologicals, somatropin and epoetin- $\alpha$ [7-9].

We investigated whether a population pharmacokinetic analysis (PPK) could also be useful in bioequivalence testing for monoclonal antibodies (MAbs), which display complex elimination mechanisms, including non-linear routes, and have a plasma half-life of one to multiple weeks. Two approaches in modelling PK data were studied. First, we developed a combined model built on all available data for both the test and reference product and tested whether adding product (test/reference) as a covariate would improve the model, indicating non-similarity. Second, we developed separate models, one for test and one for reference product. This approach does not assume similarity as a starting point and allows comparison of the model structures and parameters. For this exercise, we chose the humanised MAb trastuzumab, which targets the HER2 receptor.

\section{Methods}

\section{Study population and treatment}

Data was gathered in a phase I randomised, single-dose, parallel group bioequivalence study, preceded by a placebocontrolled dose escalation part [10]. In this study, 110 male volunteers, aged $18-45$ years, who were deemed healthy after a full medical screening, received trastuzumab in $250 \mathrm{~mL}$ $0.9 \% \mathrm{NaCl}$ as an intravenous infusion over $90 \mathrm{~min}$. Two trastuzumab products were administered: the biosimilar product (test, T), codenamed FTMB (Synthon BV, Nijmegen, The Netherlands) and the EU-licenced product (reference, R), marketed as Herceptin ${ }^{\circledR}$.

Studied dose levels of the test product in the dose escalation part were $0.5 \mathrm{mg} / \mathrm{kg}(n=6), 1.5 \mathrm{mg} / \mathrm{kg}(n=6)$ and $3 \mathrm{mg} / \mathrm{kg}$ $(n=6)$. The bioequivalence part consisted of 92 participants, who randomly received test $(n=46)$ or reference $(n=46)$ product at $6 \mathrm{mg} / \mathrm{kg}$.

Based on the trastuzumab content of the used test and reference product vials, the actual dose levels were determined to be $0.49,1.48,2.96$ and $5.96 \mathrm{mg} / \mathrm{kg}$ for $\mathrm{T}$ and $6.44 \mathrm{mg} / \mathrm{kg}$ for $\mathrm{R}$.

\section{Bioanalyses}

Trastuzumab was quantitated in serum samples collected predose and at $45 \mathrm{~min}, 1.5 \mathrm{~h}, 2 \mathrm{~h}, 3 \mathrm{~h}, 4 \mathrm{~h}, 5 \mathrm{~h}, 6 \mathrm{~h}, 8 \mathrm{~h}$ and $24 \mathrm{~h}$, and at 2, 4, 8, 14, 21, 28, 35, 42, 49 and 63 days after start of administration. A detailed description of the assay is given by Wisman [10]. The lower limit of quantification (LLOQ) was $0.060 \mu \mathrm{g} / \mathrm{mL}$. All pre-dose trastuzumab concentrations $<$ LLOQ were set to zero prior to analysis. Post-dose concentrations below LLOQ were not included. A serum sample for the quantification of serum HER2 extracellular domain (ECD) was collected prior to administration. This assay had a LLOQ of $0.50 \mathrm{ng} / \mathrm{mL}$.

In the original clinical study protocol, the sample at day 63 was not collected for PK analysis and hence not included in the previously reported NCA results [10]. However, as this sample provided valuable insight in the non-linear clearance of trastuzumab, it was included in the analyses reported in this paper.

\section{PPK}

\section{General modelling approach}

Population pharmacokinetic analysis (PPK) followed a stepwise approach. First, a general model for trastuzumab, hereafter referred to as 'combined model', was developed based on all available PK data for both test and reference product, including dose levels of the dose escalation part $(0.49,1.48$ and $2.96 \mathrm{mg} / \mathrm{kg}$ ). To investigate potential bias in the PK models due to analysing test and reference products simultaneously, PK models were also developed for the test (model $\mathrm{T}$ ) and reference product (model R) separately and included only data from subjects who were exposed to $6 \mathrm{mg} / \mathrm{kg}$ test or reference product. These are hereafter referred to as 'separate models'. The separate models were developed in parallel in order to maintain a structurally similar model for the test and reference product. Consequently, the model was only adopted if the corresponding model in the other treatment arm was preferred over its parent as well.

\section{Model development}

Model development was performed using non-linear mixed effects modelling (NONMEM 7.2.0, Icon Development Solutions, Ellicott City, MD, USA) and closely followed the 
FDA and EMA guidelines for PPK [11, 12]. Models were built under ADVAN 13 with tolerance (TOL) 9, and the first-order conditional method with interaction (FOCE-I) was used for parameter estimation. NONMEM reports an objective function value (OFV), which is the $-2 \cdot \log$ likelihood. Model hypothesis testing used the likelihood ratio test under the assumption that the difference in OFV is chi-square distributed with degrees of freedom being determined by the number of additional parameters in the more complex model. Hence, with a decrease in OFV of $\geq 7.88$ points $(p<0.005)$, the model is preferred over its parent model. Also, model performance was evaluated by means of goodness-of-fit plots, using the software package $\mathrm{R}$.

Several structural models with two or three compartments including combinations of linear and non-linear clearance were fitted to the data to determine the best structural model. Log-normal distribution of the between-subject variability $(\eta)$ was assumed, and several residual error $(\varepsilon)$ structures were tested (proportional, additive and combined).

Potential covariate correlations, defined as a significant Pearson's product-moment correlation coefficient $(p<0.01$ and $\left.r^{2}>0.5\right)$, were tested in the model development, in linear and exponential manners, and incorporated based on improvement in model performance. Explored covariates included lean body weight (LBW) [13], weight (WT), body surface area (BSA) [14], height (HT), BMI, age, HER2 ECD concentration, dose and product.

\section{Model evaluation and predictive performance}

To evaluate the robustness and predictive performance of the developed model, a visual predictive check (VPC) with 500 simulations was performed [15]. Prediction intervals of $95 \%$ were obtained by simulating the model results from the original data. Model evaluation was performed by calculating the coefficient of variation to derive the uncertainty in the parameter estimates of the model which was considered acceptable when lower than $50 \%$. Also, shrinkage, as defined by Karlsson [16], was calculated to exclude model misspecification; shrinkage less than $30 \%$ was considered acceptable.

\section{Individual pharmacokinetic profiles}

Individual pharmacokinetic profiles were simulated in $\mathrm{R}$ (version 3.2.2, $\mathrm{R}$ foundation for statistical computing, Vienna Austria) using the individual participant's model parameter estimates. Integration was performed from the start of administration until the time point when the concentration in the central compartment dropped below $0.01 \mu \mathrm{g} / \mathrm{mL}$. For the simulations, the following integration intervals were used: $1 \mathrm{~s}$ from administration until $24 \mathrm{~h}, 1 \mathrm{~min}$ until day 80 and 1 day thereafter. The concentrations were stored at original sampling times and at every $5 \mathrm{~min}$. Trastuzumab concentration at the start of administration was assumed to be $0 \mu \mathrm{g} / \mathrm{mL}$.

\section{Comparison to NCA}

For comparison to a standard NCA, AUCs were derived using model simulated (predicted) individual concentrations at the original sampling times. AUC from administration (time 0 ) to the time of the last concentration > LLOQ $\left(\mathrm{AUC}_{\text {last }}\right)$ was calculated using the linear trapezoidal method. AUC extrapolated to infinity $\left(\mathrm{AUC}_{\mathrm{inf}}\right)$ based on the apparent terminal elimination rate constant was calculated as well.

Biosimilarity statistics were performed on $\mathrm{AUC}_{\mathrm{inf}}$ or $\mathrm{AUC}_{\text {last }}$ of all participants who were exposed to $6 \mathrm{mg} / \mathrm{kg}$, comparing $\mathrm{T}$ to $\mathrm{R}$ in an unpaired $t$ test, using the software package R. AUCs were natural log (ln)-transformed prior to statistical analysis. The estimated difference in means and the corresponding $90 \%$ confidence interval (CI) were back-transformed to obtain the relative geometric mean ratio (GMR) of $\mathrm{T}$ over $\mathrm{R}(\mathrm{T} / \mathrm{R})$. These results were then compared to those calculated in a standard NCA.

To correct for the difference between actual (5.96 and $6.44 \mathrm{mg} / \mathrm{kg})$ and labelled dose $(6 \mathrm{mg} / \mathrm{kg})$, a linear normalisation to $6 \mathrm{mg} / \mathrm{kg}$ was applied to the individual AUCs in the NCA. In the PPK, individual profiles were simulated with the actual and labelled dose. Both corrected and uncorrected AUCs were calculated and statistically compared.

\section{Results}

\section{Population}

Pharmacokinetic data were gathered from 110 healthy male volunteers, whose demographics are presented in Table 1. In total, 1247 serum trastuzumab concentrations were available for the test product ( $\mathrm{T}$ ), of which 143 were <LLOQ (64 predose). In the $6 \mathrm{mg} / \mathrm{kg}$ test group, 60/906 observations were $<$ LLOQ (46 pre-dose) and for the reference product (Herceptin®), 51/912 observations (44 pre-dose).

\section{Model development}

\section{First step: combined model}

Initial exploration of the data suggested that a two- or threecompartment model would describe the data best. Based on the observed non-linear kinetics, Michaelis-Menten kinetics was incorporated, described in terms of maximum rate of elimination $\left(V_{\max }\right)$, and the concentration where $1 / 2 \cdot V_{\max }$ is reached $\left(K_{M}\right)$. Addition of a linear elimination pathway, defined by elimination rate constant $\left(k_{e}\right)$, significantly improved the model fit for both the two- and three-compartment model. 
Table 1 Demographics

\begin{tabular}{llllll}
\hline Parameter & $\begin{array}{l}\text { Test 0.5 mg/kg } \\
(n=6)\end{array}$ & $\begin{array}{l}\text { Test 1.5 mg/kg } \\
(n=6)\end{array}$ & $\begin{array}{l}\text { Test 3.0 mg/kg } \\
(n=6)\end{array}$ & $\begin{array}{l}\text { Test 6.0 mg/kg } \\
(n=46)\end{array}$ & $\begin{array}{l}\text { Reference 6.0 mg/kg } \\
(n=46)\end{array}$ \\
\hline Age (year) & $26.9(8.9)$ & $33.0(9.1)$ & $23.4(2.3)$ & $26.0(7.3)$ & $24.1(5.8)$ \\
Height $(\mathrm{cm})$ & $183(12.0)$ & $176(6.5)$ & $184(3.3)$ & $184(7.5)$ & $182(6.2)$ \\
Weight $(\mathrm{kg})$ & $73.1(12.6)$ & $73.0(8.7)$ & $72.0(7.5)$ & $79.5(11.2)$ & $77.1(10.2)$ \\
BMI $\left(\mathrm{kg} / \mathrm{m}^{2}\right)$ & $21.7(3.3)$ & $23.5(2.6)$ & $21.2(2.1)$ & $23.4(2.5)$ & $23.2(2.7)$ \\
Lean body weight $(\mathrm{kg})$ & $59.4(8.4)$ & $57.5(5.1)$ & $59.1(3.8)$ & $62.6(6.6)$ & $61.0(5.6)$ \\
Body surface area $\left(\mathrm{m}^{2}\right)$ & $1.93(0.21)$ & $1.89(0.13)$ & $1.92(0.10)$ & $2.01(0.17)$ & $1.97(0.15)$ \\
ECD $(\mu \mathrm{g} / \mathrm{L})$ & $12.7(1.8)$ & $11.8(2.1)$ & $11.4(1.5)$ & $11.3(1.8)$ & $11.8(1.8)$ \\
\hline
\end{tabular}

Mean (SD) demographics per treatment arm

$L B M$ lean body, $B S A$ body surface area, ECD HER2 extracellular domain

Adding the third compartment accounted for a delayed clearance effect. The three-compartment model, parameterised in terms of a central (V1) and peripheral volumes (V2, V3) of distribution and inter-compartmental clearances $(\mathrm{Q} 1, \mathrm{Q} 2)$, resulted in a significant improvement compared to the two-compartment model. This was confirmed by an improved goodness-of-fit, especially for the lower doses of trastuzumab. Thus, the three-compartment model was considered superior over the two compartmental models (Fig. 1). A combined residual error structure $(\varepsilon)$ proved best fit for purpose.

After identification of the structural model, individual estimates of random effects for between-subject variability were identified for the parameters $\mathrm{V} 1, K_{M}$ and $k_{e}$, with final coefficient of variation values of $14.8,35.9$ and $17.2 \%$, respectively. The residual coefficient of variation of the best model was $14.98 \%$. An omega block was required to correct for the parameter correlation between $K_{M}$ and $k_{e}$ in the model.

Significant correlations were found between lean body weight (LBW), body weight (WT), body surface area (BSA), height (HT) and body mass index (BMI) vs. V1, with correlation coefficients of $0.61,0.55,0.60,0.54$ and 0.28 , respectively. Linear regression analysis of LBW vs. BSA resulted in a coefficient of 1 and for LBW vs. WT in 0.96 . Furthermore, significant correlation coefficients were observed between BMI and $k_{e}(0.60)$, between serum concentrations HER2 ECD and $k_{e}(0.29)$, and between serum concentrations HER2 ECD and $K_{M}(0.18)$.

Implementing LBW as a linear covariate on V1 (Online Resource Eq. 1) significantly improved the objection function value (OFV) and was added to the model. Incorporating other weight-related covariates (WT, HT and BMI) separately in the model did not result in a significant improvement compared to LBW; accordingly, they were not implemented in the model. Covariate analyses identified BMI as the one most significantly correlated to $k_{e}$. Incorporating this covariate linearly on $k_{e}$ (Online Resource Eq. 2) further improved the model, and BMI was thus added to the model. Incorporating HER2 ECD as a covariate did not improve the model fit. Interestingly, the model favours lean body weight as a size descriptor to scale trastuzumab dose compared to body weight, which is used clinically in dose calculation.

Adding trastuzumab drug product (test or reference) as a covariate to the model did not explain any relevant variability. A maximum decrease in OFV of only 5.80 points $(p>0.01)$ was observed when treatment was added as a covariate on $K_{M}$.

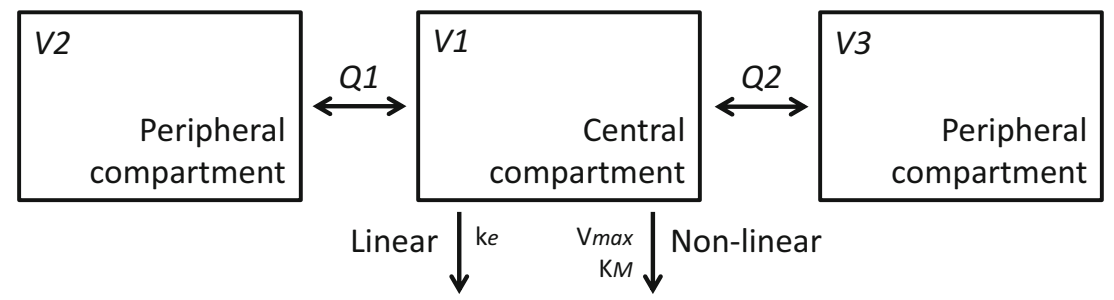

Elimination

Fig. 1 Schematic representation of the structural PK model with a parallel linear and non-linear elimination pathway. Linear elimination is described by an elimination rate constant $\left(k_{e}\right)$, and non-linear elimination is calculated as $V_{\max } C /\left(K_{M}+C\right)$ in which $V_{\max }$ is the maximum rate of elimination, $K_{M}$ is the concentration which produces half of the $V_{\max }$ and $C$ is the concentration. V1, V2 and V3 are the distribution volumes; Q1 and Q2 are the inter-compartmental clearances to the peripheral compartments 
Thus, drug product as covariate did not significantly improve model fit. All PK parameter estimates obtained with the best fit of the models are listed in Table 2.

Additionally, the rates of the linear and non-linear elimination pathway vs. serum concentration trastuzumab were calculated. At low serum concentrations of trastuzumab $(<10 \mu \mathrm{g} / \mathrm{mL})$, total elimination was almost independent of serum drug concentration, i.e. the non-linear elimination exceeded the linear elimination. At high concentrations, this pathway became saturated and the influence of non-linear elimination seemed negligible (Online Resource Fig. 3).

Also, a more complex mechanistic model approach was applied to characterise the distribution and clearance of trastuzumab: the target-mediated drug disposition (TMDD) model $[17,18]$. Besides receptor and drug-receptor complex quantification, such models are able to provide information on binding affinity of the drug to the receptor. Fitting the TMDD model to our data proved difficult due to over-parameterization. A simplified approximation TMDD model approach with a dissociation constant $K_{d}$ [19] still resulted in an incorrect fit and instability of the model, and the TMDD model approach was abandoned.

\section{Second step: separate models}

Model development of the separate models, including only data from participants who were exposed to $6 \mathrm{mg} / \mathrm{kg}$, followed a similar approach as the combined model to ensure the structural similarity. For both trastuzumab products, a third compartment could be identified, as well as a linear and a non-linear route of elimination, described by MichaelisMenten kinetics.

For the separate models, individual estimates of random effects for the between-subject variability were identified for the parameters $\mathrm{V} 1, V_{\max }$ and $k_{e}$, with final coefficient of variation values in model $\mathrm{T}$ of $16.5,12.8$ and $19 \%$, respectively. The residual coefficient of variation of the best model was $14.5 \%$. In model $\mathrm{R}$, the final coefficients of variation were $11.1,18.8$ and $17 \%$, with a residual coefficient of variation of $14.1 \%$.

Similarly to the combined model, the best model fit with the greatest reduction in OFV for both separate models was obtained by incorporating LBW as linear covariate on V1 and BMI on $k_{e}$.

\section{Model evaluation and predictive performance}

\section{Combined model}

Goodness-of-fit plots of the combined model (Fig. 2) show that all predictions lie around the line of unity. There was one outlier in the reference group, where one subject had a very low mid-infusion concentration of $0.088 \mu \mathrm{g} / \mathrm{mL}$. Virtually all
Table 2 Population PK parameters estimates from the full covariate model for trastuzumab

\begin{tabular}{clll}
\hline & Combined model & Separate model T & Separate model R \\
\hline $\begin{array}{c}\text { Parameter } \\
\text { Fixed effects }\end{array}$ & Estimate $(\mathrm{Cl})$ & & \\
V1 (L) & $3.28(3.185-3.367)$ & $3.59(3.418-3.752)$ & $3.13(3.028-3.232)$ \\
V2 (L) & $1.89(1.325-2.457)$ & $6.82(-5.572-19.21)$ & $44(28.18-59.77)$ \\
V3 $(\mathrm{L})$ & $1.96(1.736-2.179)$ & $2.15(1.858-2.443)$ & $2.09(1.929-2.244)$ \\
Q1 $\left(\mathrm{L} \mathrm{h}^{-1}\right)$ & $2.91(2.02-3.79) \times 10^{-3}$ & $2.82(1.081-4.566)$ & $3.92(3.58-4.25) \times 10^{-3}$ \\
Q2 $\left(\mathrm{L} \mathrm{h}^{-1}\right)$ & $4.34(3.66-5.01) \times 10^{-2}$ & $3.75(2.787-4.706)$ & $4.67(4.12-5.21) \times 10^{-2}$ \\
$V_{\text {max }}\left(\mu \mathrm{g} \mathrm{h}{ }^{-1}\right)$ & $178(162.3-193.1)$ & $172(138.6-205.7)$ & $127(111-143.4)$ \\
$\left.K_{M}(\mu \mathrm{g} \mathrm{L})^{-1}\right)$ & $937(759.6-1115)$ & $995(674.6-1316)$ & $1440(1189-1699)$ \\
$K_{e}\left(\mathrm{~h}^{-1}\right)$ & $2.20(2.02-2.38) \times 10^{-3}$ & $1.95(1.33-2.57) \times 10^{-3}$ & $1.76(1.62-1.9) \times 10^{-3}$ \\
Random effects & Estimate $(\mathrm{CV} \%)$ & & \\
Between-subject variability & & $0.0122(11.1)$ \\
$\omega^{2}$ V1 & $0.0217(14.8)$ & $0.0270(16.5)$ & $0.0347(18.8)$ \\
$\omega^{2} V_{\text {max }}$ & - & $0.0163(12.8)$ & - \\
$\omega^{2} K_{M}$ & $0.121(35.9)$ & - & $0.0286(17.0)$ \\
$\omega^{2} k_{e}$ & $0.0292(17.2)$ & $0.0355(19.0)$ & \\
Residual error & & & 0.0198 \\
$\sigma^{2}$ proportional & 0.0222 & 3090 & 790 \\
$\sigma^{2}$ additive & 1520 & & \\
\hline
\end{tabular}

${ }^{\text {a }}$ Explanation of parameters is given in Fig. 1

$C I$ confidence interval, $C V$ coefficient of variation, $\omega^{2}$ between-subject variance, $\sigma^{2}$ residual variance 
Fig. 2 Goodness-of-fit plots combined model. Observed vs. population predicted concentration (a), observed vs. individual predicted concentration (b), conditional weighted residuals with interaction (CWRESI) vs. time (c), and conditional weighted residuals vs population predictions (d) of the combined model
A

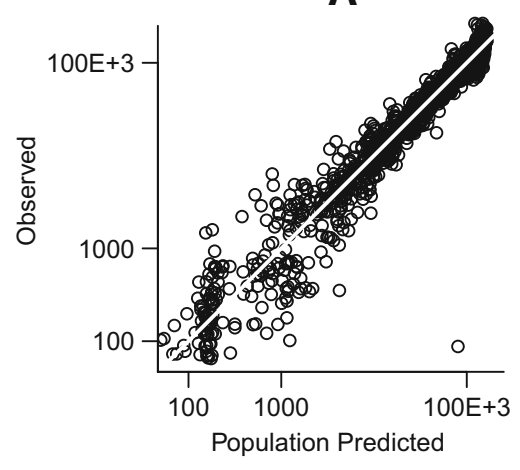

C

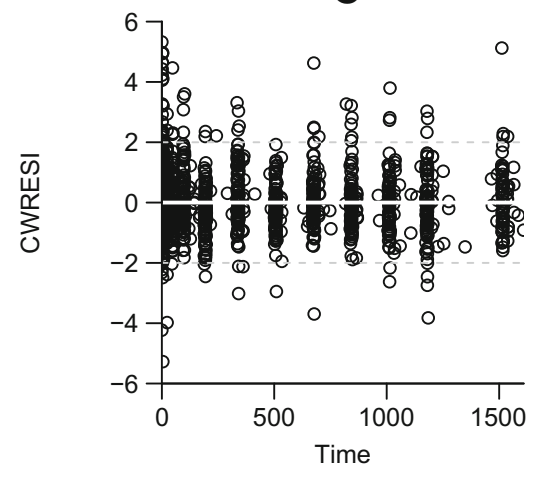

B

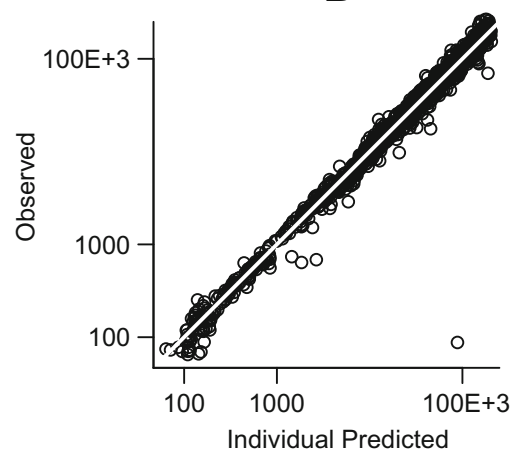

D

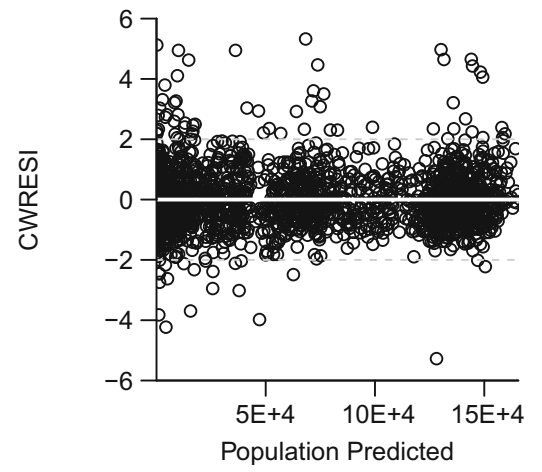

conditional weighted residuals with interaction (CWRESI) lie randomly scattered around zero without apparent bias.

The variability of the parameters V1, $K_{M}$ and $k_{e}$ on the Eta density histograms (Online Resource Fig. 4) seemed normally distributed around zero with acceptable coefficient of variation values, indicating correct description of the between-subject variability. Furthermore, no significant shrinkage was observed for parameters for which betweensubject variability was identified $(<8.04 \%)$.

The visual predictive check (VPC) proofs good predictive performance (Fig. 3) of the combined model. For the doses $>1.48 \mathrm{mg} / \mathrm{kg}$, no signs of bias were apparent and most observations lay within the $95 \%$ prediction interval (PI). Only for the lowest dose administered $(0.49 \mathrm{mg} / \mathrm{kg})$, a slightly higher prediction of the population mean was observed, especially in the lower concentration range. However, even for this dose group, most of the observations were within the $95 \%$ prediction interval.

\section{Separate models}

The goodness-of-fit plots of the separate models (Online Resource Figs. 1-2) show that predictions lie around the line of unity and that the CWRESI are observed near the central line. No bias or trend in the model prediction could be determined. The shrinkage observed for the parameters for which between-subject variability was identified $\left(\mathrm{V} 1, V_{\max }\right.$, $\left.k_{e}\right)$ is not significant $(<17.80 \%$ for model $\mathrm{T},<15.50 \%$ for model R). Additionally, the variability on the eta density histograms (Online Resource Figs. 5-6) seemed normally distributed around zero.

The population PK parameter estimates from the full covariate model is presented in Table 2. When comparing parameter estimates, most parameter distributions overlap. The parameter estimates for V2 differ between model $\mathrm{T}$ and model R, but are in the same order of magnitude. However, Q1 and Q2 for model T were higher compared to model R. In contrast to the combined model, where between-subject variability was identified for $\mathrm{V} 1, K_{M}$ and $k_{e}$, in the separate models, these were found for $\mathrm{V} 1, V_{\max }$ and $k_{e}$.

\section{Comparison to NCA}

The geometric mean (GM) AUC last obtained from the standard NCA was $1301 \mu \mathrm{g}$ day ${ }^{-1} \mathrm{~mL}^{-1}$ for the test (T) and $1588 \mu \mathrm{g} \mathrm{day}^{-1} \mathrm{~mL}^{-1}$ for the reference $(\mathrm{R})$ product. The $\mathrm{AUC}_{\text {last }}$ remained virtually unchanged when the same calculations were repeated with simulated concentrations, regardless of whether the combined model or the separate models were used (Table 3). Similar results were obtained with regard to $\mathrm{AUC}_{\text {inf. }}$.

The GM ratio (GMR) T/R with all AUC methods was 81.66-82.54\% with the lower limit (LL) of the associated $90 \%$ confidence interval (CI) below the predefined equivalence boundary of $80 \%$ (Table 3 ). Applying a linear correction to the NCA results caused the difference T-R in 
Dose $0.49 \mathrm{mg} / \mathrm{kg}$

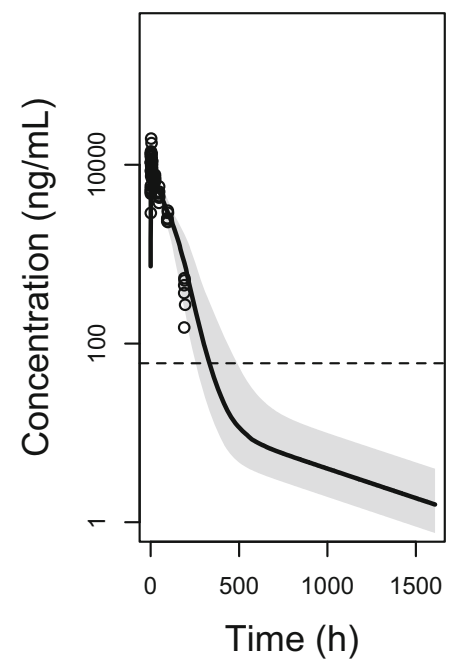

Dose $5.92 \mathrm{mg} / \mathrm{kg}$

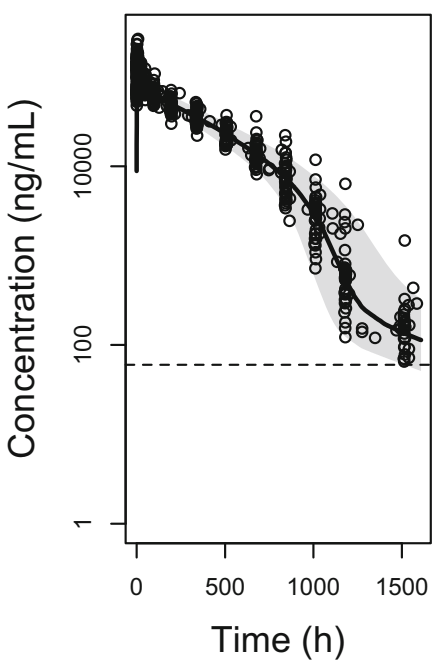

Fig. 3 Visual predictive check (VPC). Visual predictive check (VPC) of the best combined model, conditioned per dose test product $(0.49,1.48$, $2.96,5.92 \mathrm{mg} / \mathrm{kg})$ or reference product $(6.44 \mathrm{mg} / \mathrm{kg})$. The dots indicate the observations for the different trastuzumab doses administered, the

$\mathrm{AUC}_{\text {last }}$ and $\mathrm{AUC}_{\text {inf }}$ to decrease (GMR T/R 89.11-89.55\%, LL $90 \%$ CI >84.66\%). Further reductions were achieved when an equal dosage of $6 \mathrm{mg} / \mathrm{kg}$ was simulated for both trastuzumab products, which affected the AUCs in the reference product arm more profoundly and increased the GMR with approximately $2 \%$ point (Table 4 ).

Using the entire simulated profile, as opposed to only the simulated concentrations at the original sampling times, generally resulted in a small decrease of $1-2 \%$ compared with the NCA for both $\mathrm{AUC}_{\text {last }}$ and $\mathrm{AUC}_{\text {inf }}$, with the exception of the $\mathrm{AUC}_{\mathrm{inf}}$ calculated on profiles derived with model $\mathrm{R}$, where an average increase of $1.7 \%$ was observed (Online Resource Tables 1 and 2). Conversely, with the combined
Dose $2.96 \mathrm{mg} / \mathrm{kg}$

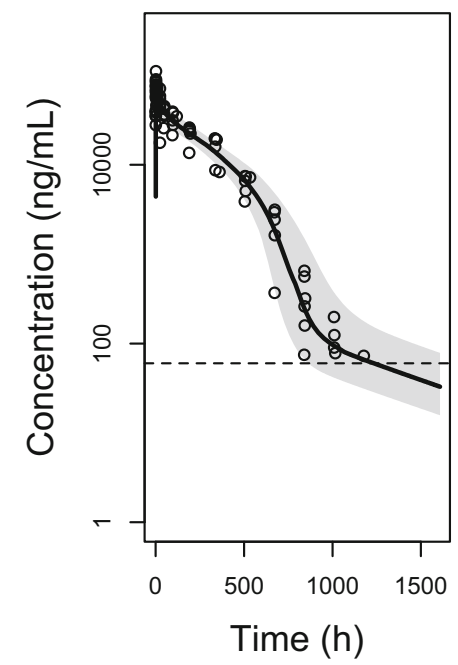

All Doses

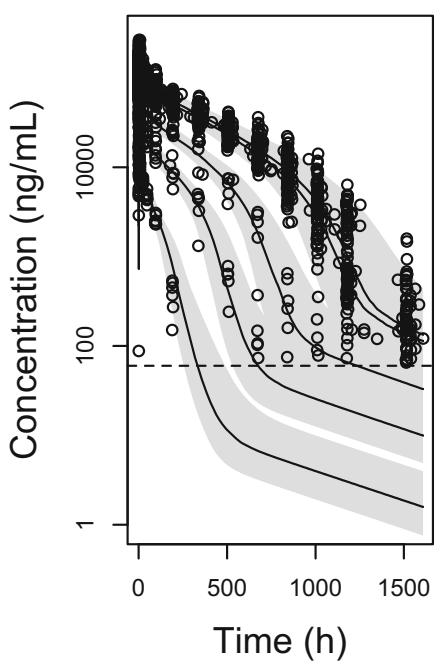

lines are the typical predicted concentrations by the model for each dose and the grey area is the $95 \%$ prediction interval (PI). The dotted line is the assay's lower limit of quantification (LLOQ) for trastuzumab $(0.060 \mu \mathrm{g} / \mathrm{mL})$

model, lower AUCs were obtained compared with the separate models for T only.

\section{Discussion}

As long as generic products are being developed, controversy and scepticism regarding the claims of therapeutic equality have followed marketed bioequivalent products. Recently, Bate et al. [20] advocated that for the more complex pharmaceuticals, two allegedly bioequivalent drug products may not be interchangeable, which could have adverse consequences. MAbs are certainly among the most complex 
Table 3 AUC comparison actual dose

\begin{tabular}{llll}
\hline & GM & & GM ratio (\%) \\
\hline AUC ${ }_{\text {last }}$ & Test & Reference & T/R \\
NCA & 1301 & 1588 & $81.91(77.82-86.22)$ \\
Separate models & 1300 & 1588 & $81.86(78.08-85.82)$ \\
$\quad$ Combined model & 1296 & 1588 & $81.59(77.88-85.47)$ \\
AUC inf $_{\text {NCA }}$ & & & \\
Separate models & 1311 & 1593 & $82.32(78.17-86.69)$ \\
Combined model & 1300 & 1591 & $82.54(78.70-86.57)$ \\
\hline
\end{tabular}

Geometric mean (GM) ( $\mu \mathrm{g}$ day $\left.{ }^{-1} \mathrm{~mL}^{-1}\right)$ and GM ratio (\%) with the $90 \%$ confidence for the actual dose (test $5.92 \mathrm{mg} / \mathrm{kg}$; reference $6.44 \mathrm{mg} / \mathrm{kg}$ ) as derived by different methods per treatment arm

$T$ test, $R$ reference

therapeutics, and establishing similarity to the reference product can thus be challenging.

For demonstrating pharmacokinetic biosimilarity in a human population, a NCA is virtually always performed and its results (AUC and $C_{\max }$ ) compared statistically, even though it is widely recognised that the NCA is less suitable for drugs with complex non-linear kinetics, as is the case for MAbs.

Population approach pharmacokinetic (PK) modelling and simulation techniques have been successfully applied to quantitatively describe the PK of MAbs in humans [21-26]. Such an approach has been applied in bioequivalence studies and also for biotherapeutics [9], where it was found to give indistinguishable results on the standard NCA parameters (AUC and $\mathrm{C}_{\max }$ ), as was the case in our analysis. However, as was also argued by Dubois et al. [9], a PK model can provide valuable insight in the biological systems underlying the PK properties. Although the standard NCA-derived parameters, such as $\mathrm{C}_{\max }, \mathrm{AUC}_{\mathrm{inf}}$, terminal half-life, etc.,

Table 4 AUC comparison after dose correction

\begin{tabular}{llll}
\hline & GM & & GM ratio (\%) \\
\hline AUC ${ }_{\text {last }}$ & Test & Reference & T/R \\
NCA & 1318 & 1479 & $89.11(84.66-93.79)$ \\
Separate models & 1323 & 1455 & $90.93(86.72-95.35)$ \\
$\quad$ Combined model & 1319 & 1443 & $91.41(87.25-95.76)$ \\
AUC & & & \\
NCA & 1329 & 1484 & $89.55(85.03-94.30)$ \\
Separate models & 1337 & 1457 & $91.74(87.46-96.24)$ \\
Combined model & 1324 & 1446 & $91.54(87.37-95.92)$ \\
\hline
\end{tabular}

Geometric mean (GM) ( $\mu \mathrm{g}$ day $\left.{ }^{-1} \mathrm{~mL}^{-1}\right)$ and GM ratio (\%) with the $90 \%$ confidence for the labelled dose $(6 \mathrm{mg} / \mathrm{kg})$ as derived by different methods per treatment arm. For the NCA results, a linear dose correction was applied; in the models, the labelled dose was used to simulate the individual profiles (see main body)

$T$ test, $R$ reference may seem similar, the two drug products could behave quite differently in terms of PK, a feature that goes undetected in a NCA [27]. Furthermore, similar plasma concentrations do not invariably mean similar concentrations at the site of action.

Here, we describe two methods of incorporating PK modelling in biosimilarity research. The first approach is developing a model on all available data from both test and reference product(s) and carefully examining possible bias in one of the treatment groups. Testing for (statistically) significant differences between drug product can be done for all the model parameters via covariate analysis. Covariate testing follows a well-established statistical distribution that can be used for statistical inference [28, 29]. If no significant correlations can be identified between the drug products and if attempts to incorporate treatment as covariate in the model fail to improve it, the biosimilarity claim is supported.

The second method entails the development of different models, one for each test and reference product(s), which in contrast to a combined model does not assume similarity between test and reference product as a starting point. This method allows comparison of the model structure that should be identical for biosimilar products and of model parameters for both test and reference product.

Comparing different PK models inevitably reveals minor differences for which the clinical significance needs to be discussed. For example, in model $\mathrm{T}$, the optimal inter-compartmental clearances (Q1 and Q2) were estimated to be a factor $10^{2}-10^{3}$ higher than the corresponding parameters in the other models, while the striking dissimilarity did not seem to affect the descriptive properties of the overall profiles. However, as the (fictive) second and third compartments were not sampled, this finding merely reflects a mathematical solution to a rather complex problem and not necessarily a true (e.g. physiological or pharmacological) difference. Additionally, the higher dose administered for the reference product could have allowed a better characterisation of the terminal portion of the PK profile (elimination parameters), which also affects the estimation of remaining parameters such as Q1 and Q2.

This represents an important limitation of the second method, which may be of particular relevance when modelling PK data from two different populations separately. Unfortunately, pharmacokinetic biosimilarity of biotherapeutics is regularly investigated in trials of parallel design, because of the long half-life and the potential of antidrug antibodies development, which could influence the pharmacokinetics [30]. Theoretically, all MAbs share common pharmacokinetic properties, e.g. small central volume of distribution, no renal excretion due to large molecular size, metabolism into amino acids and peptides, both specific (non-linear) and non-specific (linear) cellular uptake and degradation elimination mechanisms [31-35]. Thus, the remaining variability is probably determined by 
patient characteristics. When comparing the model parameters of the separate models, one of the most prominent differences is the population estimate for V1, which is unlikely caused by a difference between test and reference product.

The combined model equally well described the data, without bias in either the test or reference group. Adding trastuzumab drug product as covariate to the model could not explain any residual variability, which not only strongly supports the biosimilarity claim but also indicates that the difference in AUCs must be attributed to population characteristics.

From a regulatory perspective, another limitation of the second method is the lack of proper statistical inference testing on the model parameters. One might consider overlapping confidence intervals for parameter estimates indications for biosimilarity, but many parameters are related, so that for example a low inter-compartmental clearance may be 'compensated' in the model by a low volume of distribution. An extension of 'bioequivalence statistics' has been applied to model parameters by Wilkens et al. [36], although their method suffers from the aforementioned limitations as well.

Notwithstanding the limitations of PPK, it has several benefits over a NCA. Importantly, a PPK is not concerned with differences in administered doses. Although EMA allows a dose correction in the bioequivalence guideline (for chemically-derived products) if the difference exceeds $5 \%$, the NCA assumes linearity in its correction, which is not appropriate for MAbs, that display non-linear pharmacokinetics. Other benefits of PPK include the possibility to identify and thus correct for certain covariates and the relative robustness of a PPK against protocol deviations, with regard to timing of sample collection, missing samples, duration of intravenous administration and incomplete administration $[8,37]$.

Simulations with model $\mathrm{R}$ revealed that the two allowed extremes for protein content per batch (effective dose 5.28 and $7.2 \mathrm{mg} / \mathrm{kg}$ ) would result in a $90 \% \mathrm{CI}$ for the GMR for $\mathrm{AUC}_{\text {last }}$ of $146.39-147.22 \%$ in a cross-over design $(n=46)$. If such batch-to-batch variations are not considered relevant, then the consequences on the standard biosimilarity parameters may also be argued to be irrelevant.

With a PK model, multiple scenarios can be simulated within these extremes, which can be used to build the case that the test product achieves therapeutic drug concentrations, similar to the reference product, when administered according to a certain dosing regimen. This approach also circumvents some of the aforementioned limitations of direct comparison of two or more models. If a biomarker or pharmacological effect can be measured in the biosimilarity trial and incorporated in a pharmacokinetic-pharmacodynamic model (pharmacodynamic model), a relevant clinical target may be simulated and lend further support to a biosimilarity claim.

The NCA will most likely remain a gold standard in biosimilarity research, even for the complex MAbs.
Nonetheless, the model approach can serve as an elegant add-on. Questions that need to be addressed before a PPK can fully substitute the NCA in demonstrating biosimilarity relate to selection of the most meaningful PK or pharmacodynamic parameter from the model, and the minimal population size to detect with sufficient statistical power relevant (model) differences.

Previously, the benefits of modelling and simulation have been proposed for proof of biosimilarity, to which this paper adds similar benefits for MAbs.

Author Contributions JR, JB and JS designed the research; TvD and JS performed the research; JR, JS, TvD and JB analysed the data; FS contributed to the development of the PK models. All authors contributed to the writing of the manuscript.

Compliance with ethical standards All procedures performed in studies involving human participants were in accordance with the ethical standards of the institutional and/or national research committee and with the 1964 Helsinki declaration and its later amendments or comparable ethical standards.

Conflict of interest The authors declare that they have no conflict of interest.

Open Access This article is distributed under the terms of the Creative Commons Attribution 4.0 International License (http:// creativecommons.org/licenses/by/4.0/), which permits unrestricted use, distribution, and reproduction in any medium, provided you give appropriate credit to the original author(s) and the source, provide a link to the Creative Commons license, and indicate if changes were made.

\section{References}

1. Bonafede M, Joseph GJ, Princic N, Harrison DJ (2013) Annual acquisition and administration cost of biologic response modifiers per patient with rheumatoid arthritis, psoriasis, psoriatic arthritis, or ankylosing spondylitis. J Med Econ 16:1120-1128

2. Francis SM, Heyliger A, Miyares MA, Viera M (2015) Potential cost savings associated with dose rounding antineoplastic monoclonal agents. J Oncol Pharm Pract 21:280-284

3. IMS health. IMS Health Top 20 Global Products 2014; 2015. http://www.imshealth.com/files/web/Corporate/News/Top-Line Market Data/2014/Top_20_Global_Products_2014.pdf. Accessed 1 April 2016.

4. WHO Expert Committee on Biological Standardization. Guidelines on evaluation of similar biotherapeutic products (SBPs). Geneva: 2009.

5. US Food and Drug Administration. Scientific considerations in demonstrating biosimilarity to a reference product. Silver Spring: 2015.

6. Committee for Medicinal Products for Human Use (CHMP). Guideline on similar biological medicinal products. London: 2015.

7. Panhard X, Mentré F (2005) Evaluation by simulation of tests based on non-linear mixed-effects models in pharmacokinetic interaction and bioequivalence cross-over trials. Stat Med 24: $1509-1524$

8. Dubois A, Gsteiger S, Pigeolet E, Mentré F (2010) Bioequivalence tests based on individual estimates using non-compartmental or model-based analyses: evaluation of estimates of sample means and type I error for different designs. Pharm Res 27:92-104 
9. Dubois A, Gsteiger S, Balser S, Pigeolet E, Steimer JL, Pillai G, Mentré F (2012) Pharmacokinetic similarity of biologics: analysis using nonlinear mixed-effects modeling. Clin Pharmacol Ther 91: 234-242

10. Wisman LAB, De Cock EPM, Reijers JAA, Kamerling IMC, Van Os SHG, de Kam ML, Burggraaf J, Voortman G (2014) A phase I dose-escalation and bioequivalence study of a trastuzumab biosimilar in healthy male volunteers. Clin Drug Investig 34:887-894

11. US Food and Drug Administration. Population pharmacokinetics. Silver Spring: 1999.

12. Committee for Medicinal Products for Human Use (CHMP). Guideline on reporting the results of population pharmacokinetic analyses. London: 2008.

13. Janmahasatian S, Duffull SB, Ash S, Ward LC, Byrne NM, Green B (2005) Quantification of lean bodyweight. Clin Pharmacokinet 44: 1051-1065

14. Mosteller RD (1987) Simplified calculation of body-surface area. N Engl J Med 317:1098

15. Post TM, Freijer JI, Ploeger BA, Danhof M (2008) Extensions to the visual predictive check to facilitate model performance evaluation. J Pharmacokinet Pharmacodyn 35:185-202

16. Karlsson MO, Savic RM (2007) Diagnosing model diagnostics. Clin Pharmacol Ther 82:17-20

17. Luu KT, Bergqvist S, Chen E, Hu-Lowe D, Kraynov E (2012) A model-based approach to predicting the human pharmacokinetics of a monoclonal antibody exhibiting target-mediated drug disposition. J Pharmacol Exp Ther 341:702-708

18. Mager DE, Jusko WJ (2001) General pharmacokinetic model for drugs exhibiting target-mediated drug disposition. J Pharmacokinet Pharmacodyn 28:507-532

19. Gibiansky L, Gibiansky E, Kakkar T, Ma P (2008) Approximations of the target-mediated drug disposition model and identifiability of model parameters. J Pharmacokinet Pharmacodyn 35:573-591

20. Bate R, Mathur A, Lever HM, Thakur D, Graedon J, Cooperman T, Mason P, Fox ER (2015) Generics substitution, bioequivalence standards, and international oversight: complex issues facing the FDA. Trends Pharmacol Sci 37:184-191

21. Bruno R, Washington CB, Lu J-F, Lieberman G, Banken L, Klein P (2005) Population pharmacokinetics of trastuzumab in patients with HER2+ metastatic breast cancer. Cancer Chemother Pharmacol 56:361-369

22. Charoin J-E, Jacqmin P, Banken L, Lennon S, Jorga K. Population pharmacokinetic analysis of trastuzumab (Herceptin) following long-term administration using different regimens. PAGE 2004; 13:Abstr 489.

23. Fukushima Y, Charoin J-E, Brewster M, Jonsson EN. Population pharmacokinetic analysis of trastuzumab
(Herceptin $\left({ }^{\circledR}\right)$ based on data from three different dosing regimens. PAGE 2007; 16:Abstr 1121.

24. Kloft C, Graefe E-U, Tanswell P, Scott AM, Hofheinz R, Amelsberg A, Karlsson MO (2004) Population pharmacokinetics of sibrotuzumab, a novel therapeutic monoclonal antibody, in cancer patients. Investig New Drugs 22:39-52

25. Kuester K, Kovar A, Lüpfert C, Brockhaus B, Kloft C (2009) Refinement of the population pharmacokinetic model for the monoclonal antibody matuzumab: external model evaluation and simulations. Clin Pharmacokinet 48:477-487

26. van Hasselt JGC, Boekhout AH, Beijnen JH, Schellens JHM, Huitema ADR (2011) Population pharmacokineticpharmacodynamic analysis of trastuzumab-associated cardiotoxicity. Clin Pharmacol Ther 90:126-132

27. Rescigno A, Powers J, Herderick EE (2001) Bioequivalent or nonbioequivalent? Pharmacol Res 43:543-547

28. Khandelwal A, Harling K, Jonsson EN, Hooker AC, Karlsson MO (2011) A fast method for testing covariates in population PK/PD models. AAPS J 13:464-472

29. Meibohm B, Derendorf H (1997) Basic concepts of pharmacokinetic/pharmacodynamic (PK/PD) modelling. Int $\mathrm{J}$ Clin Pharmacol Ther 35:401-413

30. Committee for Medicinal Products for Human Use (CHMP). Guideline on similar biological medicinal products containing monoclonal antibodies: non-clinical and clinical issues. London: 2012.

31. Wang W, Wang EQ, Balthasar JP (2008) Monoclonal antibody pharmacokinetics and pharmacodynamics. Clin Pharmacol Ther 84:548-558

32. Tabrizi MA, Tseng CL, Roskos LK (2006) Elimination mechanisms of therapeutic monoclonal antibodies. Drug Discov Today 11:81-88

33. Ternant D, Bejan-Angoulvant T, Passot C, Mulleman D, Paintaud G (2015) Clinical pharmacokinetics and pharmacodynamics of monoclonal antibodies approved to treat rheumatoid arthritis. Clin Pharmacokinet 54:1107-1123

34. Dirks NL, Meibohm B (2010) Population pharmacokinetics of therapeutic monoclonal antibodies. Clin Pharmacokinet 49:633-659

35. Keizer RJ, Huitema ADR, Schellens JHM, Beijnen JH (2010) Clinical pharmacokinetics of therapeutic monoclonal antibodies. Clin Pharmacokinet 49:493-507

36. Wilkins JJ, Gautier A, Lowe PJ. Bioequivalence, bootstrapping and case-deletion diagnostics in a biologic: a model-based analysis of the effect of formulation differences in a monoclonal antibody. PAGE 2008; 17:Abstr 1284.

37. Charles B (2014) Population pharmacokinetics: an overview. Aust Prescr 37:210-213 